\title{
ELECTROSTATIC IMAGE THEORY FOR TWO INTERSECTING CONDUCTING SPHERES
}

\author{
I. V. Lindell, K. H. Wallén, and A. H. Sihvola \\ Electromagnetics Laboratory \\ Helsinki University of Technology \\ Otakaari 5A, 02015HUT Espoo 15, Finland
}

\begin{abstract}
The classical electrostatic image principle for a perfectly conducting object consisting of two orthogonally intersecting spheres is revisited through vector analysis. A method for finding the image of a given charge distribution by splitting it in three virtual singlesphere problems is described. Also, a simple relation between the image charges of a given point charge is found. The method is applied to finding exact expressions for the polarizability dyadic of the object to be used as a bechmark for testing computing schemes on a nontrivial geometry. Application of the present object as a model for an oblong particle in a composite dielectric is also discussed.
\end{abstract}

\section{Introduction}

\section{Intersecting Spheres}

2.1 Image Sources

2.2 Method of Virtual Spheres

2.3 Vanishing Total Charge

2.4 Polarizability

\section{Special Cases}

3.1 Axial Excitation

3.2 Transverse Excitation

\section{Applications}

\section{Conclusion}

\section{References}




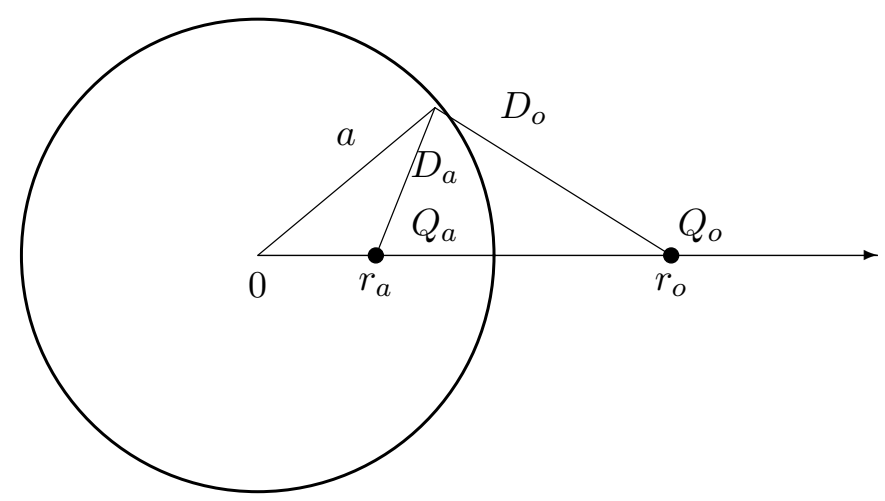

Figure 1. Kelvin's image principle for a point charge $Q_{o}$ in front of a PEC sphere at zero potential can be seen from the geometry.

\section{INTRODUCTION}

The image principle offers one of the simplest methods to solving boundary-value problems both in static and dynamic electromagnetics. The problem of a point charge $Q_{o}$ at the distance $r_{o}$ from the center of a perfectly conducting (PEC) sphere of radius $a$ was solved by William Thomson (later Lord Kelvin) as a young Cambridge graduate in 1845 [1]. He showed that the potential could be solved in replacing the sphere by a single point charge $Q_{a}$ located at a certain distance $r_{a}$, Figure 1. The principle can be simply found from the geometry. In fact, because the potential is inversely proportional to the distance from the charge point, if the potential created by the charge $Q_{o}$ on the spherical surface should equal the negative of that from the image charge $Q_{a}$, the ratio of the distances $D_{o}$ and $D_{a}$ should be constant for all points on the surface. From Figure 1 it is seen that this is so if the two triangles with the same angle at the center of the sphere are similar in which case their corresponding sides must be in the proportion $a / r_{o}$ whence $r_{a}=a^{2} / r_{o}$. The total potential vanishes when the image charge is chosen in the same proportion with opposite sign, $Q_{a}=-\left(a / r_{o}\right) Q$.

This simple principle led Thomson to a transformation (later called the Kelvin transformation) which states that if a potential function $\phi(\mathbf{r})$ is a solution to the Poisson equation with the charge density function $\varrho(\mathbf{r})$ as its source,

$$
\nabla^{2} \phi(\mathbf{r})=-\frac{1}{\epsilon} \varrho(\mathbf{r})
$$


the transformed potential and source functions

$$
\phi_{K}(\mathbf{r})=\frac{a}{r} \phi\left(\mathbf{r}_{K}\right), \quad \varrho_{K}(\mathbf{r})=\frac{a^{5}}{r^{5}} \varrho\left(\mathbf{r}_{K}\right)
$$

also satisfy the Poisson equation for the same $\epsilon$ [2] with

$$
\mathbf{r}_{K}(\mathbf{r})=\frac{a^{2}}{r^{2}} \mathbf{r}
$$

For the proof see, e.g., [3]. Because PEC boundary conditions remain valid in the transformation, it gave Thomson a method to find a great number of solutions to new boundary-value problems through transformations of known solutions to old problems. For example, the problem of a point charge between two PEC planes making an angle $\pi / n$ for $n=1,2,3 \ldots$, solvable in terms of $2 n-1$ image charges, could be transformed to one with two PEC spheres intersecting at the same angle, as shown by Thomson already in 1845 [1]. Since then, the basic case $n=2$ corresponding to two orthogonally intersecting spheres has been treated in many books [2,5-8] as well as articles, e.g., [9-12]. Because the approach in all of these references is based on the Kelvin transformation, bipolar coordinates or some other more exotic principle, it appears worth while to rederive the solution in terms of the basic Kelvin's image principle. Using vector calculus, this can be done with little effort.

\section{INTERSECTING SPHERES}

Let us now consider the problem of a PEC object made of two intersecting spheres of radii $a$ and $b$. Let the axis of symmetry coincide with the $z$ axis and the plane of intersection coincide with the $x y$ coordinate plane. The centers of the spheres are defined by the respective vectors $\mathbf{d}_{a}=-\mathbf{u}_{z} d_{a}, \mathbf{d}_{b}=\mathbf{u}_{z} d_{b}$, Figure $2 . \mathbf{u}_{z}$ is the unit vector in the positive $z$ direction and $\mathbf{d}=-\mathbf{d}_{a}+\mathbf{d}_{b}$ denotes the vector between the centers of the spheres. One can show that if the image of a given point charge consists of three point charges, the spheres must intersect orthogonally. This is also obvious from the Kelvin inversion of an orthogonal PEC corner. Assuming orthogonal spheres we have from the geometry of Figure 2

$$
d_{a}=\frac{a^{2}}{d}, \quad d_{b}=\frac{b^{2}}{d}, \quad d=d_{a}+d_{b}=\sqrt{a^{2}+b^{2}}
$$

The radius $c$ of the circle of intersection is

$$
c=\frac{a b}{d}=\frac{a b}{\sqrt{a^{2}+b^{2}}}=\sqrt{d_{a} d_{b}} .
$$




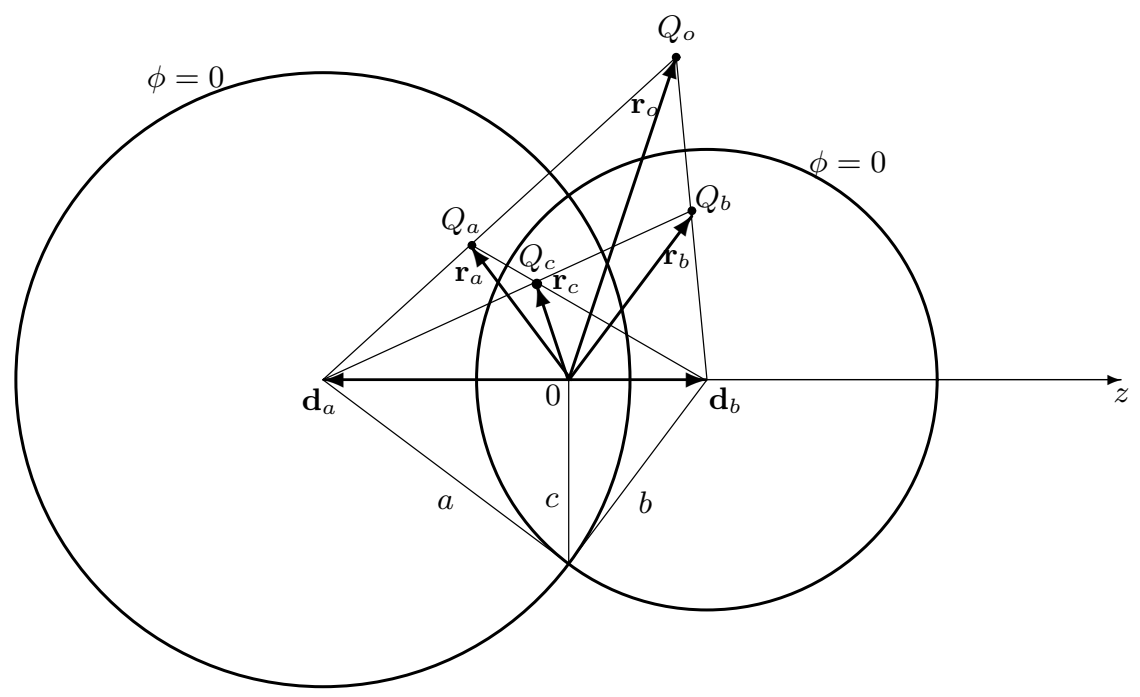

Figure 2. Image problem of a conducting object made of two orthogonally intersecting spheres. The exciting point charge $Q_{o}$ and three image charges $Q_{a}, Q_{b}, Q_{c}$ in free space make zero potential on the surface of the object.

\subsection{Image Sources}

The original charge $Q_{o}$ is assumed to be at the point $\mathbf{r}_{o}$ outside the object. It is now required that the potential from this charge at the surface of the double sphere is canceled by that from three image charges $Q_{a}, Q_{b}, Q_{c}$ located at the respective points $\mathbf{r}_{a}, \mathbf{r}_{b}, \mathbf{r}_{c}$.

The image charges and their locations are found by applying the image principle four times, twice for both spherical surfaces, to produce zero potential on the spherical surfaces. The potential is zero on the a-sphere if $Q_{a}$ is required to be the image of $Q_{o}$, and $Q_{c}$ the image of $Q_{b}$, with respect to the a-sphere. On the other hand, the potential is zero on the b-sphere if $Q_{b}$ is required to be the image of $Q_{o}$, and $Q_{c}$ the image of $Q_{a}$, with respect to the b-sphere. In this way the images $Q_{a}$ and $Q_{b}$ become fully defined. For $Q_{c}$ there are two conditions which are compatible when the spheres are orthogonal.

Starting from the images $Q_{a}$ and $Q_{b}$, their respective points $\mathbf{r}_{a}$ and $\mathbf{r}_{b}$ can be easily found by temporarily shifting the origin to the sphere centers:

$$
\mathbf{r}_{a}-\mathbf{d}_{a}=\frac{a^{2}}{\left|\mathbf{r}_{o}-\mathbf{d}_{a}\right|^{2}}\left(\mathbf{r}_{o}-\mathbf{d}_{a}\right), \quad \mathbf{r}_{b}-\mathbf{d}_{b}=\frac{b^{2}}{\left|\mathbf{r}_{o}-\mathbf{d}_{b}\right|^{2}}\left(\mathbf{r}_{o}-\mathbf{d}_{b}\right),
$$




$$
Q_{a}=-\frac{a}{\left|\mathbf{r}_{o}-\mathbf{d}_{a}\right|} Q_{o}, \quad Q_{b}=-\frac{b}{\left|\mathbf{r}_{o}-\mathbf{d}_{b}\right|} Q_{o} .
$$

The main problem is to find the third image $Q_{c}$ and its location $\mathbf{r}_{c}$. Let us first define it as the image of $Q_{b}$ in the a-sphere. The condition for the image location $\mathbf{r}_{c}$ is

$$
\mathbf{r}_{c}-\mathbf{d}_{a}=\frac{a^{2}}{\left|\mathbf{r}_{b}-\mathbf{d}_{a}\right|^{2}}\left(\mathbf{r}_{b}-\mathbf{d}_{a}\right)
$$

Inserting from (6)

$$
\mathbf{r}_{b}-\mathbf{d}_{a}=\frac{b^{2}}{\left|\mathbf{r}_{o}-\mathbf{d}_{b}\right|^{2}}\left(\mathbf{r}_{o}-\mathbf{d}_{b}\right)+\mathbf{d}
$$

and expanding through some algebraic steps

$$
\left|\mathbf{r}_{b}-\mathbf{d}_{a}\right|^{2}=\frac{r_{o}^{2} d^{2}}{\left|\mathbf{r}_{o}-\mathbf{d}_{b}\right|^{2}}
$$

we obtain

$$
\mathbf{r}_{c}-\mathbf{d}_{a}=\frac{a^{2} b^{2}}{r_{o}^{2} d^{2}}\left(\mathbf{r}_{o}-\mathbf{d}_{b}\right)+\frac{a^{2}\left|\mathbf{r}_{o}-\mathbf{d}_{b}\right|^{2}}{r_{o}^{2} d^{2}} \mathbf{d} .
$$

From this we can solve the location of the image $Q_{c}$ as

$$
\mathbf{r}_{c}=\frac{a^{2} b^{2}}{d^{2} r_{o}^{2}}\left(\mathbf{r}_{o}-2 \mathbf{u}_{z} \mathbf{u}_{z} \cdot \mathbf{r}_{o}\right)=\frac{c^{2}}{r_{o}^{2}} \mathbf{r}_{o}^{\prime}, \quad \mathbf{r}_{o}^{\prime}=\left(\overline{\overline{\mathbf{I}}}-2 \mathbf{u}_{z} \mathbf{u}_{z}\right) \cdot \mathbf{r}_{o}
$$

The dyadic $\overline{\overline{\mathbf{I}}}-2 \mathbf{u}_{z} \mathbf{u}_{z}$ has the property of reflecting any vector $\mathbf{q}$ to its mirror image $\mathbf{q}^{\prime}=\mathbf{q}-2 \mathbf{u}_{z}\left(\mathbf{u}_{z} \cdot \mathbf{q}\right)$ with respect to the plane of intersection $z=0$. Thus, $r_{o}^{2}=r_{o}^{\prime 2}$.

The magnitude of the image $Q_{c}$ is obtained as

$$
Q_{c}=-\frac{a}{\left|\mathbf{r}_{b}-\mathbf{d}_{a}\right|} Q_{b}=\frac{a b}{\left|\mathbf{r}_{b}-\mathbf{d}_{a}\right|\left|\mathbf{r}_{o}-\mathbf{d}_{b}\right|} Q_{o}=\frac{a b}{r_{o} d} Q_{o}=\frac{c}{r_{o}^{\prime}} Q_{o} .
$$

Since the expressions (12), (13) obtained for the charge $Q_{c}$ and its location $\mathbf{r}_{c}$ are symmetric functions of $a$ and $b$, the result does not change if we consider it as the image of $Q_{a}$ in the b-sphere. Thus, the two sets of conditions are compatible.

\subsection{Method of Virtual Spheres}

From the previous results we see that the three images of the point source $Q_{o}$ at $\mathbf{r}_{o}$ in the PEC object can be found very simply through 


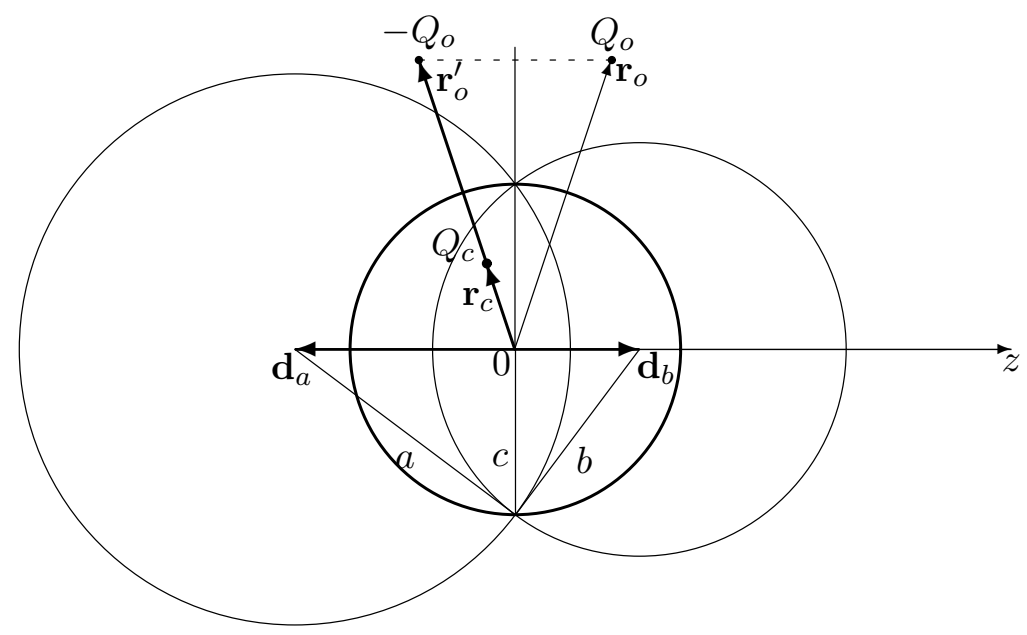

Figure 3. The third image charge $Q_{c}$ and its location $\mathbf{r}_{c}$ for two orthogonally intersecting PEC spheres can be found simply by applying Kelvin's image principle to a virtual origocentric PEC sphere defined by the circle of intersection with radius $c$. The original charge $Q_{o}$ is replaced by the virtual source $-Q_{o}$ at $\mathbf{r}_{o}^{\prime}$ which is the mirror image of $\mathbf{r}_{o}$ in the plane of intersection.

the basic Kelvin image principle. The image $Q_{a}$ and its location $\mathbf{r}_{a}$ are found as the Kelvin image of the original source when only the PEC a-sphere is present. Similarly, the image $Q_{b}$ and its location $\mathbf{r}_{b}$ are found when only the PEC b-sphere is present. From (12) and (13) we see that the third image $Q_{c}$ and its location $\mathbf{r}_{c}$ are found as the image of $-Q_{o}$ at the mirror image location $\mathbf{r}_{o}^{\prime}=\mathbf{r}_{o}-2 \mathbf{u}_{z}\left(\mathbf{u}_{z} \cdot \mathbf{r}_{o}\right)$ in a virtual origocentric PEC sphere with radius $c=a b / \sqrt{a^{2}+b^{2}}$, Figure 3 . Although the idea of the third PEC sphere with the radius $a b / d$ has been referred to previously [12], the procedure for the image source could not be found from the available literature. However, the method of virtual PEC spheres gives a convenient method to find the images of any given charge density function $\varrho(\mathbf{r})$ as a combination of three image sources, Figure 4,

$$
\begin{gathered}
\varrho_{a}(\mathbf{r})=-\frac{a^{5}}{r^{5}} \varrho\left(\frac{a^{2}}{r^{2}} \mathbf{r}\right), \\
\varrho_{b}(\mathbf{r})=-\frac{b^{5}}{r^{5}} \varrho\left(\frac{b^{2}}{r^{2}} \mathbf{r}\right), \\
\varrho_{c}(\mathbf{r})=\frac{c^{5}}{r^{5}} \varrho\left(\frac{c^{2}}{r^{2}}\left(\overline{\overline{\mathbf{I}}}-2 \mathbf{u}_{z} \mathbf{u}_{z}\right) \cdot \mathbf{r}\right) .
\end{gathered}
$$




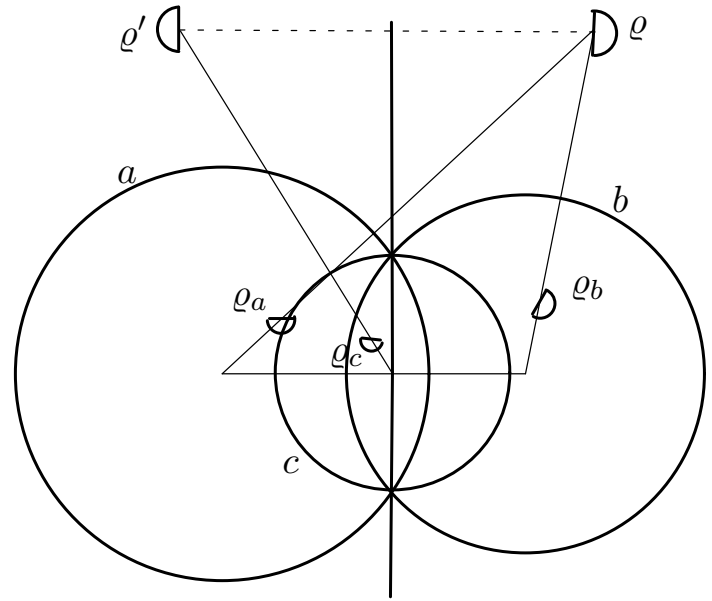

Figure 4. Method of virtual spheres gives the image of a charge density function $\varrho(\mathbf{r})$ as a sum of three image functions. $\varrho_{c}$ is the image of reflected source $\varrho^{\prime}(\mathbf{r})$ in the virtual PEC sphere of radius $c=a b / d$.

This method can also be understood through the Kelvin transformation which maps the two spheres to two orthogonal planes. Of the three images in the transform space the two negative ones are obtained as images in the two planes. The third positive charge cannot be obtained as an image in a single PEC plane but it needs two steps, being an image of an image. Now this is not a unique procedure and the two PEC planes can be chosen in infinitely many ways. Choosing one of the planes so that it transforms to a plane, the process is simplified in the sphere problem because, instead of finding two images in spheres, one has to take a simple image in a plane (mirror reflection with sign change) and just one image in a virtual sphere. Two plane reflections could be reduced to one by choosing a virtual PMC plane with the Neumann condition $\mathbf{n} \cdot \nabla \phi=0$. However, a PMC plane is not transformed to a PMC sphere but to a sphere with a special impedance boundary condition. This method of virtual spheres can obviously be generalized to two spheres intersecting at any angle $\pi / n$ and the resulting images can be obtained either in one virtual PEC sphere (the negative images) or through a reflection in a PEC plane and as an image in a virtual PEC sphere (the positive images). The generalization will be considered in a forthcoming paper.

There is also an interesting relation between the four point 
charges,

$$
\frac{1}{Q_{a}^{2}}+\frac{1}{Q_{b}^{2}}=\frac{1}{Q_{c}^{2}}+\frac{1}{Q_{o}^{2}},
$$

which can be derived from the expressions (7) and (13). It can be used as a check after finding the image charges. Such a relation is also valid in the limiting case $Q_{o} \rightarrow \infty$ and $r_{o} \rightarrow \infty$ for the three charges $Q_{a}, Q_{b}, Q_{c}$ on the $z$ axis making constant potential at the surface of the object. Because a similar formula can also be derived between the five axial point charges associated with the problem of two PEC spheres intersecting at an angle $\pi / 3$, it can be a special case of a more universal relation. (17) may have some novelty since it could not be found from the literature available to these authors. Its physical significance is still an open question.

\subsection{Vanishing Total Charge}

If the original charge $Q_{o}$ recedes to infinity, $r_{o} \rightarrow \infty$, the image charges vanish as $1 / r_{o}$. If simultaneously the charge grows $Q_{o} \rightarrow \infty$ so that $Q_{o} / r_{o}$ is finite, the potential from $Q_{o}$ at the object is constant,

$$
\phi_{o} \rightarrow \frac{Q_{o}}{4 \pi \epsilon_{o} r_{o}} .
$$

The image charges then become finite

$$
Q_{a} \rightarrow-\frac{a Q_{o}}{r_{o}}, \quad Q_{b} \rightarrow-\frac{b Q_{o}}{r_{o}}, \quad Q_{c} \rightarrow \frac{c Q_{o}}{r_{o}}
$$

and their locations are

$$
\mathbf{r}_{a} \rightarrow \mathbf{d}_{a}, \quad \mathbf{r}_{b} \rightarrow \mathbf{d}_{b}, \quad \mathbf{r}_{c} \rightarrow 0
$$

This kind of charges create a potential which cancels $\phi_{o}$ at the surface of the object. More generally, charges of the form

$$
Q_{a}=\alpha a, \quad Q_{b}=\alpha b, \quad Q_{c}=-\alpha c,
$$

positioned on the $z$ axis as (20), create a potential which has a constant value on the surface of the two spheres,

$$
\phi_{\alpha}=\frac{\alpha}{4 \pi \epsilon_{o}} .
$$

Thus, the capacitance of the object is $[2,4-7]$

$$
C=\frac{Q_{a}+Q_{b}+Q_{c}}{\phi_{\alpha}}=4 \pi \epsilon_{o}(a+b-c)=4 \pi \epsilon_{o}\left(a+b-\frac{a b}{\sqrt{a^{2}+b^{2}}}\right) .
$$


In the original problem the aim was to find image sources in a grounded PEC object, i.e., an object whose potential is zero. This requires that the total charge in the object

$$
Q_{t}=Q_{a}+Q_{b}+Q_{c}=-\frac{a Q_{o}}{\left|\mathbf{r}_{o}-\mathbf{d}_{a}\right|}-\frac{b Q_{o}}{\left|\mathbf{r}_{o}-\mathbf{d}_{b}\right|}+\frac{c}{r_{o}} Q_{o}
$$

is not zero and its value depends on the original charge and its location. If we wish to have zero total charge on the object, the charge $-Q_{t}$ must be added so that the potential on the surface of the object is constant. This means that the factor $\alpha$ in (21) must be chosen as

$$
\alpha=-\frac{Q_{t}}{a+b-c}
$$

and the balancing charges must be positioned as defined by (20).

\subsection{Polarizability}

The PEC object made of two intersecting spheres can be used as a model for a non-spherical inclusion in a mixture and its polarization properties are of interest. For a dilute mixture the distance between the objects is large enough so that we can approximate the local electric field by a constant vector $\mathbf{E}_{o}$. The field from the original point charge $Q_{o}$ at the origin is

$$
\mathbf{E}_{o}=-\frac{Q \mathbf{r}_{o}}{4 \pi \epsilon_{o} r_{o}^{3}}
$$

when the distance is large, $r_{o} \gg a, b$. When the obstacle has no net charge, its effect can be approximated by a dipole moment $\mathbf{p}$, which depends on the local field through a polarization dyadic $\overline{\bar{\alpha}}$,

$$
\mathbf{p}=\overline{\bar{\alpha}} \cdot \mathbf{E}_{o}
$$

Let us define a numerical parameter, the relative polarizability $\overline{\bar{\alpha}}_{r}$ by

$$
\overline{\bar{\alpha}}_{r}=\frac{\overline{\bar{\alpha}}}{\epsilon_{o} V},
$$

where $V$ is the volume of the obstacle. The expression for the volume of a spherical cap given in [14], p. 315, subtracted from the volume of the a-sphere gives

$$
V_{a}=\frac{4 \pi a^{3}}{3}\left(\frac{1}{2}+\frac{3 a}{4 d}-\frac{a^{3}}{4 d^{3}}\right)
$$




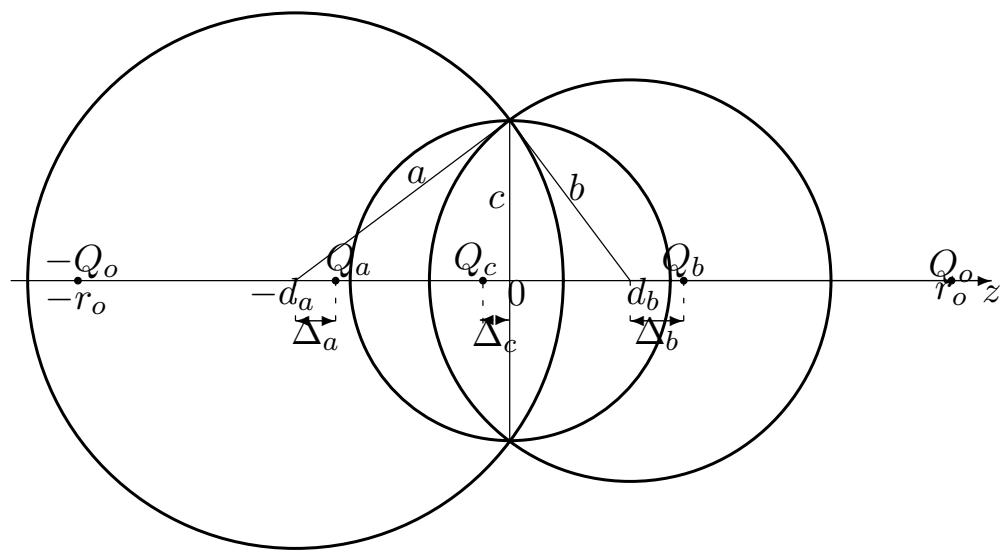

Figure 5. Axial excitation of the PEC object. The virtual source $-Q_{o}$ giving the image charge $Q_{c}$ lies now at the point $\mathbf{r}_{o}^{\prime}=-\mathbf{r}_{o}=-\mathbf{u}_{z} r_{o}$.

and similarly for the b-sphere. In the symmetric case $a=b, d=2 c=$ $a \sqrt{2}$, the total volume becomes

$$
V=V_{a}+V_{b}=\frac{8+5 \sqrt{2}}{8} \frac{4 \pi a^{3}}{3} \approx 1.884 \frac{4 \pi a^{3}}{3} .
$$

Because of rotational symmetry of the object, the polarizability dyadic consists of axial and transverse components,

$$
\overline{\bar{\alpha}}=\mathbf{u}_{z} \mathbf{u}_{z} \alpha_{z}+\left(\overline{\bar{I}}-\mathbf{u}_{z} \mathbf{u}_{z}\right) \alpha_{t},
$$

and, similarly, the relative polarizabilities are $\alpha_{r z}, \alpha_{r t}$. The two scalar components can be found by considering two special cases for the original charge point $\mathbf{r}_{o}$.

\section{SPECIAL CASES}

\subsection{Axial Excitation}

Let us consider the case when the original charge $Q_{o}$ lies on the $z$ axis, $\mathbf{r}_{o}=\mathbf{u}_{z} r_{o}$. Because of symmetry, all images also lie on the $z$ axis and their locations differ from the centers of the spheres as

$$
\begin{array}{cc}
\mathbf{r}_{a}=-\mathbf{u}_{z}\left(d_{a}-\Delta_{a}\right), & \Delta_{a}=\frac{a^{2}}{r_{o}+d_{a}} \\
\mathbf{r}_{b}=\mathbf{u}_{z}\left(d_{b}+\Delta_{b}\right), & \Delta_{b}=\frac{b^{2}}{r_{o}-d_{b}}
\end{array}
$$




$$
\mathbf{r}_{c}=-\mathbf{u}_{z} \Delta_{c}, \quad \Delta_{c}=\frac{c^{2}}{r_{o}} .
$$

The corresponding image charges are

$$
Q_{a}=-\frac{a Q_{o}}{r_{o}+d_{a}}, \quad Q_{b}=-\frac{b Q_{o}}{r_{o}-d_{b}}, \quad Q_{c}=\frac{c Q_{o}}{r_{o}} .
$$

The total image charge becomes

$$
Q_{t}=-\frac{a Q_{o}}{r_{o}+d_{a}}-\frac{b Q_{o}}{r_{o}-d_{b}}+\frac{c Q_{o}}{r_{o}} .
$$

In the symmetric case the problem becomes simpler. Substituting

$$
b=a, \quad d=a \sqrt{2}, \quad d_{b}=d_{a}=c=a / \sqrt{2},
$$

the image locations and charges are now defined by

$$
\begin{gathered}
\Delta_{a}=\frac{a^{2}}{r_{o}+a / \sqrt{2}}, \quad \Delta_{b}=\frac{a^{2}}{r_{o}-a / \sqrt{2}}, \quad \Delta_{c}=\frac{a^{2}}{2 r_{o}}, \\
Q_{a}=-\frac{a Q_{o}}{r_{o}+a / \sqrt{2}}, \quad Q_{b}=-\frac{a Q_{o}}{r_{o}-a / \sqrt{2}}, \quad Q_{c}=\frac{a Q_{o}}{r_{o} \sqrt{2}} .
\end{gathered}
$$

The dipole moment due to these image charges with respect to the origin is

$$
\begin{aligned}
\mathbf{p} & =\mathbf{u}_{z}\left(d_{a}-\frac{a^{2}}{r_{o}+d_{a}}\right) \frac{a Q_{o}}{r_{o}+d_{a}}-\mathbf{u}_{z}\left(d_{a}+\frac{a^{2}}{r_{o}-d_{a}}\right) \frac{a Q_{o}}{r_{o}-d_{a}}-\mathbf{u}_{z} c \frac{d_{a}^{2} Q_{o}}{r_{o}^{2}} \\
& =\mathbf{u}_{z} p_{z} .
\end{aligned}
$$

The total image charge is now

$$
Q_{t}=-\frac{2(2 \sqrt{2}-1) r_{o}^{2}+a^{2}}{\sqrt{2} r_{o}\left(2 r_{o}^{2}-a^{2}\right)} a Q_{o} .
$$

The balancing charge $-Q_{t}$ is distributed symmetrically according to (21), (25) as

$$
Q_{b}=Q_{a}=-\frac{1}{7}(4+\sqrt{2}) Q_{t}, \quad Q_{c}=-Q_{a} / \sqrt{2}=\frac{1}{7}(2 \sqrt{2}+1) Q_{t} .
$$


However, because of symmetry, it does not contribute to the dipole moment at the origin. Letting the charge recede to infinity, $r_{o} \rightarrow \infty$, the magnitude of the axial dipole moment (40) approaches the limit

$$
p_{z} \rightarrow-\frac{1}{4}(12+\sqrt{2}) \frac{a^{3} Q_{o}}{r_{o}^{2}} .
$$

The axial polarizability $\alpha_{r z}$ can now be computed from (28) by inserting the electric far field expression (26)

$$
\mathbf{E}_{o}=-\mathbf{u}_{z} \frac{Q_{o}}{4 \pi \epsilon_{o} r_{o}^{2}}
$$

and the volume (30) to give

$$
\alpha_{r z}=\frac{6}{7}(43-26 \sqrt{2}) \approx 5.340383467 .
$$

This result gives a useful benchmark for testing the accuracy of numerical computation schemes for an object with nontrivial geometry. Forming the integral equation for the surface charge density on the object and using the boundary-element method with high-order basis functions, a numerical computation has been made which reproduced the first four digits of (45), [15]. Same result was obtained by evaluating numerically an integral expression given in [13].

\subsection{Transverse Excitation}

Let us consider another special case with the point charge $Q_{o}$ lying at the plane of intersection outside the two orthogonally intersecting spheres, $\mathbf{r}_{o} \cdot \mathbf{d}_{a}=\mathbf{r}_{o} \cdot \mathbf{d}_{b}=0$, Figure 6 . In this case the virtual source $-Q_{o}$ stays at the original source point $\mathbf{r}_{o}$ and, thus, the image $Q_{c}$ lies in the $x y$ plane.

In the symmetric case $a=b$ the images are

$$
\begin{aligned}
Q_{a}=Q_{b} & =-\frac{a \sqrt{2}}{\sqrt{2 r_{o}^{2}+a^{2}}} Q_{o}, \quad r_{a}=r_{b}=\frac{a^{2} \sqrt{2}}{\sqrt{2 r_{o}^{2}+a^{2}}}, \\
Q_{c} & =\frac{c}{r_{o}} Q_{o}=\frac{a}{r_{o} \sqrt{2}} Q_{o}, \quad r_{c}=\frac{c^{2}}{r_{o}}=\frac{a^{2}}{2 r_{o}} .
\end{aligned}
$$

Because of symmetry, for transverse excitation the dipole moment is transverse to the $z$ axis. Like in the axial excitation case, the balancing 


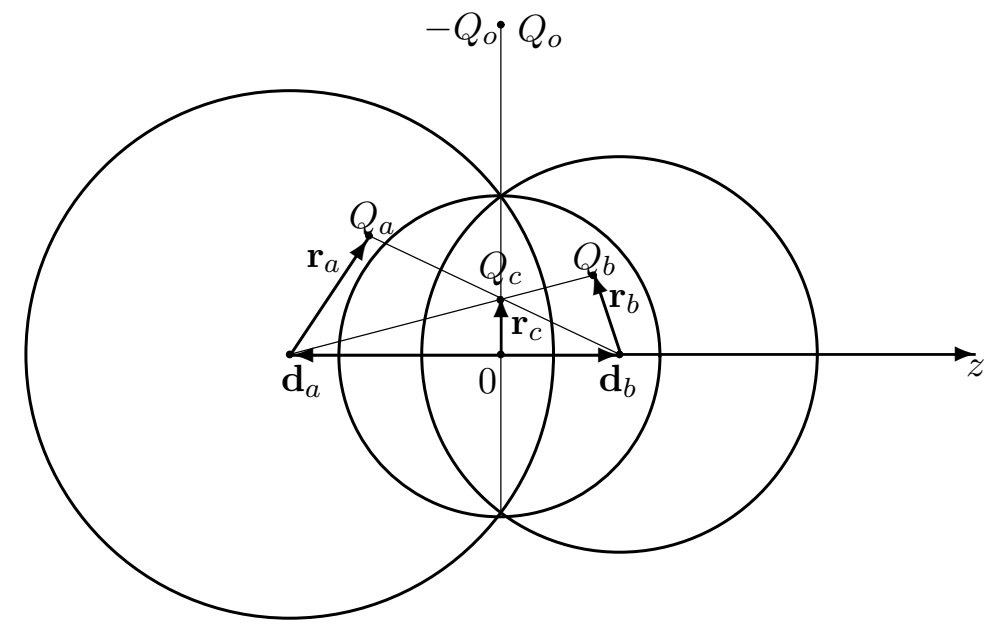

Figure 6. Image problem of a conducting object made of two orthogonally intersecting spheres when the exciting point charge $Q_{o}$ lies on the plane of intersection. The virtual source $-Q_{o}$ lies at the point of $Q_{o}$ and the image charge $Q_{c}$ lies on the plane of intersection.

charge on the $z$ axis does not contribute to the polarization moment. From the geometry or $r_{o} \rightarrow \infty$ the transverse dipole moment becomes

$$
p_{t} \rightarrow-\frac{2 a^{3} Q_{o}}{r_{o}^{2}}+\frac{c^{3} Q_{o}}{r_{o}^{2}}=-\frac{1}{4}(8-\sqrt{2}) \frac{a^{3} Q_{o}}{r_{o}^{2}} .
$$

Normalized this gives the transverse polarizability

$$
\alpha_{r t}=\frac{\left|p_{t}\right|}{\epsilon_{o}\left|E_{t}\right| V}=\frac{4 \pi r_{o}^{2}\left|p_{t}\right|}{Q_{o} V}=\frac{6}{7}(37-24 \sqrt{2}) \approx 2.6218924,
$$

which, like (45), could be used as a benchmark [15].

\section{APPLiCATIONS}

In many applications of electromagnetics, the polarizability and dipole moments of material particles play a very essential role. This is the case, for example, in the study of wave interaction and scattering from heterogeneous media in remote sensing, geophysical, and nondestructive measurement applications, and in the design of composites and complex metamaterials $[16,17]$.

The electromagnetic response of a mixture where inclusions are embedded in host medium is strongly determined by the polarizability 
of the inclusions. In addition to contributing to the effective permittivity of the material [18], also the absorption characteristics of the composite are dependent on the polarizabilities. This is especially the case when metallic inclusions are mixed into nonconducting host material with the objective of enhancing the response of the medium. The imaginary part of the permittivity of the composite describes the effective absorption of the homogenized mixture. Metallic inclusions have also an important use in percolating mixtures [19] where small changes in the volume fractions of the components or other structural parameters may lead to strong variations in macroscopic response.

Consider a mixture where conducting inclusions are randomly distributed within a host (background) material. Let the inclusions be of the double-sphere shape that has been treated in the previous sections. For simplicity, let the permittivity of the background material be $\epsilon_{o}$ and the number density of the inclusion particles $n$. The effective permittivity of the mixture can be calculated according to the Maxwell Garnett theory [20]. Because the double spheres are not spherically symmetric, the macroscopic response of the mixture depends on the orientation distribution of the inclusions. In the extreme case all inclusions are aligned in the global coordinate system, and then the mixture is uniaxially anisotropic with the optical axis along the rotation axis of the inclusions. On the other hand, the case where the orientation distribution is random, the effective medium is isotopic. In this case the effective permittivity of the mixture is

$$
\epsilon_{\mathrm{eff}}=\epsilon_{o}+\frac{n \alpha_{\mathrm{av}}}{1-n \alpha_{\mathrm{av}} /\left(3 \epsilon_{o}\right)}
$$

where the average polarizability is

$$
\alpha_{\mathrm{av}}=\frac{1}{3} \operatorname{tr}\{\overline{\bar{\alpha}}\}=\frac{\alpha_{z}+2 \alpha_{t}}{3}
$$

For dilute mixtures, the effective permittivity reads

$$
\epsilon_{\mathrm{eff}} / \epsilon_{o} \approx 1+n \alpha_{\mathrm{av}} / \epsilon_{o}=1+f \frac{\alpha_{r z}+2 \alpha_{r t}}{3}
$$

and here $f=n V$ is the volume fraction of the inclusions. Inserting the values for the polarizability components ((45) and (49)) for the double sphere, we have $\epsilon_{r} \approx 1+3.53 f$. This means that the effective susceptibility is about 18 percent higher than in the case if the inclusions were metal spheres having the same volume fraction. In the spherical-inclusion case, the relative permittivity would be $\epsilon_{r} \approx 1+3 f$. The spherical geometry is known to be an extreme shape in the sense 
that any deviation from it only can increase the average polarizability of the inclusion [21].

This type of Maxwell Garnett model for a mixture with metallic sphere doublets is the first approximation. For denser mixtures where the inclusions are in a more near-field contact with each other, the disturbing field of one inclusion on the neighbor is no longer homogeneous. But then the interaction effect and induced dipole due to the inhomogeneous incidence can be modelled by applying the image principles of Section 2 where a point-source excitation was allowed to be anywhere in the neighborhood of the inclusion.

\section{CONCLUSION}

Image theory was reviewed for a perfectly conducting object made of two orthogonally intersecting spheres. Instead of applying the Kelvin transformation, the simple Kelvin image principle and vector analysis was used to arrive at the image expressions. It was shown that the three image charges of a given point charge can be found through a very straightforward method of virtual spheres which applies only the single-sphere image method to three PEC spheres and can be applied to any given original charge distribution. For a point charge $Q_{o}$, two of the images are Kelvin images in each of the two PEC spheres. The third image is obtained as the Kelvin image of a virtual charge $-Q_{o}$ at another point in space in a virtual PEC sphere whose radius equals that of the circle of intersection. In the analysis a simple relation between the original and image point charges was found which appears to be a special case of a more general relation. Axial and transverse polarizabilities of the PEC object set in a homogeneous field were obtained which, being exact results, can be used to test the accuracy of numerical procedures. Finally, use of the object as a model for an inclusion in a composite material was discussed.

\section{REFERENCES}

1. Thomson, W. (Kelvin), "Extrait d'une lettre de M. William Thomson á M. Liouville," originally in Journal de Mathematiques Pures et Appliqués, Vol. 10, 364, 1845; Vol. 12, 256-264, 1847. Reprint of Papers on Electricity and Magnetism, Macmillan, London, 144-146, 1872.

2. Maxwell, J. C., A Treatise on Electricity and Magnetism, 3rd ed., Vol. 1, Clarendon Press 1891, reprint Dover, 261-263, 1954.

3. Lindell, I. V. and J. J. Hänninen, "Static image principle for the 
sphere in isotropic or bi-isotropic space," Radio Sci., Vol. 35, No. 3, 653-660, May-June 2000.

4. Thomson, J. J., Elemente der Mathematischen Theorie der Elektrizität und Magnetismus, Chapter 5, Vieweg, Brunswick, 1897.

5. Kottler, F., "Elektrostatik der Leiter," Handbuch der Physik, W. Westphal (ed.), Vol. 12, 420-422, 434-436, Springer, Berlin, 1927.

6. Jeans, J., The Mathematical Theory of Electricity and Magnetism, 5th ed., Chapter 8, University Press, Cambridge, 1933.

7. Smythe, W. R., Static and Dynamic Electricity, 2nd ed., 125, McGraw-Hill, New York, 1950.

8. Lebedev, N. N., I. P. Skalskaya, and Y. S. Uflyand, Worked Problems in Applied Mathematics, 242-247, Dover, New York, 1979.

9. McAllister, I. W., "On the electrostatics of spherical protrusions," IEE Proc. A, Vol. 133, No. 2, 84-88, 1986.

10. Kim, I. C. and S. Torquato, "Effective conductivity of suspensions of overlapping spheres," J. Appl. Phys., Vol. 71, No. 6, 2727-2735, March 1992.

11. Zhou, H.-X., A. Szabo, J. F. Douglas, and J. B. Hubbard, "A Brownian dynamics algorithm for calculating the hydrodynamic friction and the electrostatic capacitance of an arbitrary shaped object," J. Chem. Phys., Vol. 100, No. 5, 3821-3826, March 1994.

12. Palaniappan, D. and B. U. Felderhof, "Electrostatics of the conducting double sphere," J. Appl. Phys., Vol. 86, No. 6, 34183422, September 1999.

13. Felderhof, B. U. and D. Palaniappan, "Longitudinal and transverse polarizability of the conducting double sphere," $J$. Appl. Phys., Vol. 88, No. 9, 4947-4952, November 2000.

14. Zwillinger, D. (ed.), CRC Standard Mathematical Tables and Formulae, 30th ed., CRC Press, Boca Raton, 1996.

15. Sihvola, A. H. and P. Ylä-Oijala, work to be published.

16. Tsang, L., J. A. Kong, and R. T. Shin, Theory of microwave remote sensing, Wiley, New York, 1986.

17. Milton, G. W., The Theory of Composites, Cambridge University Press, 2002.

18. Sihvola, A., Electromagnetic Mixing Formulas and Applications, IEE Publishing, London, Electromagnetic Waves Series 47, 1999.

19. Stauffer, D. and A. Aharony, Introduction to percolation theory, Second edition, Taylor \& Francis, London and Philadelphia, 1994. 
20. Maxwell Garnett, J. C., "Colours in metal glasses and in metal films", Trans. Royal Society (London), Vol. CCIII, 385-42, 1904.

21. Schiffer, M. and G. Szegö, "Virtual mass and polarization", Transactions of the American Mathematical Society, Vol. 67, Issue 1, 130-205, September 1949.

Ismo V. Lindell was born in Viipuri, Finland, in 1939. He received the Dr.Tech. (Ph.D.) degree at Helsinki University of Technology (HUT), Espoo, Finland, in 1971. Currently he is a Professor of electromagnetic theory at the Electromagnetics Laboratory at the HUT. During 1996-2001 he also held the research position of Professor of the Academy of Finland. Dr. Lindell has authored and coauthored over 200 scientific papers and 11 books, for example, Methods for Electromagnetic Field Analysis (IEEE Press, New York, 2nd ed., 1995), Electromagnetic Waves in Chiral and Bi-Isotropic Media (Artech House, Norwood MA, 1994) and History of Electrical Engineering (Otatieto, Espoo, Finland 1994, in Finnish). Dr. Lindell received the IEEE S.A. Schelkunoff price (1987) and the IEE Maxwell Premium (1997 and 1998). Since 2002, he is a Guest Professor of Southeast University, Nanjing China.

Henrik Wallén was born in 1975. He received the M.Sc. (Tech.) degree in Electrical Engineering in 2000 from Helsinki University of Technology (HUT), Espoo, Finland. He is currently doing his postgraduate studies working at the HUT Electromagnetics Laboratory. His main research interests include electromagnetic theory and computational methods.

Ari Henrik Sihvola was born on October 6th, 1957, in Valkeala, Finland. He received the degrees of Diploma Engineer in 1981, Licentiate of Technology in 1984, and Doctor of Technology in 1987, all in Electrical Engineering, from the Helsinki University of Technology, Finland. Besides working for HUT and the Academy of Finland, he was visiting engineer in the Research Laboratory of Electronics of the Massachusetts Institute of Technology, Cambridge, in 1985-1986, and in 1990-1991, he worked as a visiting scientist at the Pennsylvania State University, State College. In 1996, he was visiting scientist at the Lund University, Sweden, and for the academic year 20002001 he was visiting professor at the Electromagnetics and Acoustics Laboratory of the Swiss Federal Institute of Technology, Lausanne. Ari Sihvola is professor of electromagnetics in Helsinki University of Technology with interest in electromagnetic theory, complex media, 
materials modelling, remote sensing, and radar applications. Ari Sihvola is Vice Chairman of the Finnish National Committee of URSI (International Union of Radio Science). He also served as the Secretary of the 22nd European Microwave Conference, held in August 1992, in Espoo, Finland. 